\title{
A nonperturbative quantum electrodynamic approach to the theory of laser induced high harmonic generation
}

\author{
Chao $\mathbf{Y u}^{1}$, Jingtao Zhang ${ }^{2}$, Zhi-Wei Sun ${ }^{3}$, Zhenrong Sun ${ }^{1, *}$, Dong-Sheng Guo ${ }^{1,4, \dagger}$ \\ ${ }^{1}$ State Key Laboratory of Precision Spectroscopy, Department of Physics, East China Normal University, Shanghai 200062, China \\ ${ }^{2}$ State Key Laboratory of High-field Laser Physics, Shanghai Institute of Optical and Fine Mechanics, \\ Chinese Academy of Sciences, Shanghai 201800, China \\ ${ }^{3}$ Key Laboratory of Microgravity, Institute of Mechanics, Chinese Academy of Sciences, Beijing 100190, China \\ ${ }^{4}$ School of Physics, Peking University, Beijing 100871, China \\ Corresponding authors.E-mail: *zrsun@phy.ecnu.edu.cn, ${ }^{\dagger} d s g u o p h d @ 163 . c o m$ \\ Received April 2, 2014; accepted May 6, 2014
}

\begin{abstract}
Using nonperturbative quantum electrodynamics, we develop a scattering theory for high harmonic generation (HHG). A transition rate formula for HHG is obtained. Applying this formula, we calculate the spectra of high harmonics generated from different noble gases shined by strong laser light. We study the cutoff property of the spectra. The data show that the cutoff orders of high harmonics are greater than that predicted by the "3.17" cutoff law. As a numerical experiment, the data obtained from our repeated calculations support the newly derived theoretical expression of the cutoff law. The cutoff energy of high harmonics described by the new cutoff law, in terms of the ponderomotive energy $U_{p}$ and the ionization potential energy $I_{p}$, is $3.34 U_{p}+1.83 I_{p}$. The higher cutoff orders predicted by this theory are due to the absorption of the extra photons, which participate only the photon-mode up-conversion and do nothing in the photoionization process.
\end{abstract}

Keywords high harmonic generation (HHG), cutoff law, strong laser physics, nonperturbative quantum electrodynamics, transition rate formula

PACS numbers 32.80.Rm, 42.65.Ky, 12.20.Ds, 03.65.Nk

\section{Introduction}

Laser induced high harmonic generation (HHG) from noble gases has attracted people's attentions since the first observation in 1987 [1]. Many experimental and theoretical studies have been done over the last three decades. The HHG spectra carry information not only from the laser fields, but also from the atoms of gas media. The information extracted from atoms may be used in the tomographic reconstruction of atoms [2]. The observed high harmonics were of odd orders. Different electron recombination models were developed to account for HHG spectra. Most of the models were in the category of semiclassical theories. A notable one among those theories was a so-called three-step model $[3,4]$. It successfully derived a cutoff law $q_{c} \hbar \omega=3.17 U_{p}+1.32 I_{p}$ for the $\mathrm{HHG}$ energy. In the expression of the cutoff law, $U_{p}$ is the pondermotive energy, $I_{p}$ the ionization potential energy, $\omega$ the laser photon frequency, and $q_{c}$ the cutoff order of HHG. There were also some nonperturbative quantum electrodynamics (NPQED) theories to account for phenomena in HHG with some artificial assumptions [5-7]. HHG is a very complicated physical process. New findings from new experiments in this area are still booming up. But all semiclassical and NPQED theories with artificial assumptions cannot truly account for all physical subprocesses. To correctly reveal all the physical subprocesses and to give experimental measurements better interpretations, it is necessary to develop a rigorous NPQED theoretical approach to HHG.

In a very recent experiment, ultrahigh-order harmonics were observed, up to orders greater than 5000 [8]. These high orders look going beyond the cutoff order predicted by the "3.17" law. To interpret this new experimental result, re-deriving HHG transition formula becomes an immediate need. With the transition rate formula, one can study the features of HHG and the cutoff law.

In this paper, we derive the HHG transition rate formula strictly according to the NPQED theory [9-10]. In this approach, quantum-field Volkov states are used as 
intermediate states, followed by an electron transition back to the original atomic bound state [12]. With the derived transition rate formula, we calculate HHG spectra for $\mathrm{He}, \mathrm{Ne}, \mathrm{Ar}, \mathrm{Kr}$, Xe atoms in different laser beam intensities and wavelengths. In our calculations, all allowed photon-induced electron transitions are included. We find that photons participating mode up-conversion are not only those ionization photons. There are also some photons which participate the mode up-conversion, but do not participate the photoionization.

\section{The rate formula of high harmonic generation}

We consider a quantized laser field of frequency $\omega$ corresponding to a wavevector $\boldsymbol{k}$. The harmonic mode, treated as a spontaneous emission, is of frequency $\omega^{\prime}$ and a wavevector $\boldsymbol{k}^{\prime}$. In this paper, we use $\hbar=1, c=1$, and $e=-|e|$. In the Schrödinger picture, the Hamiltonian of the atom-radiation interaction system is [13]

$$
H=H_{0}+U(\boldsymbol{r})+V+V^{\prime},
$$

where

$$
H_{0}=\frac{(-\mathrm{i} \nabla)^{2}}{2 m_{e}}+\omega N_{a}+\omega^{\prime} N_{a}^{\prime}
$$

is the noninteraction part of the Hamiltonian. Here, $N_{a}$ and $N_{a}^{\prime}$ are photon number operators of the laser and the spontaneously emitted photon mode, respectively. And $U(\boldsymbol{r})$ is the atomic binding potential, $m_{e}$ the electron rest mass. The total electron-photon interaction is $V+V^{\prime}$ with

$$
\begin{aligned}
V= & -\frac{e}{2 m_{e}}[\boldsymbol{A}(\boldsymbol{r}) \cdot(-\mathrm{i} \nabla)+(-\mathrm{i} \nabla) \cdot \boldsymbol{A}(\boldsymbol{r})]+\frac{e^{2} \boldsymbol{A}(\boldsymbol{r})^{2}}{2 m_{e}} \\
V^{\prime}= & -\frac{e}{2 m_{e}}\left[\boldsymbol{A}^{\prime}(\boldsymbol{r}) \cdot(-\mathrm{i} \nabla)+(-\mathrm{i} \nabla) \cdot \boldsymbol{A}^{\prime}(\boldsymbol{r})\right] \\
& +\frac{e^{2} \boldsymbol{A}(\boldsymbol{r}) \cdot \boldsymbol{A}^{\prime}(\boldsymbol{r})}{m_{e}},
\end{aligned}
$$

where $\boldsymbol{A}(\boldsymbol{r})$ and $\boldsymbol{A}^{\prime}(\boldsymbol{r})$ denote the vector potential of the laser photon mode and the spontaneously emitted photon mode, respectively. We use $\boldsymbol{A}(\boldsymbol{r})=g\left(\epsilon \mathrm{e}^{\mathrm{i} \boldsymbol{k} \cdot \boldsymbol{r}} a+\right.$ $\left.\boldsymbol{\epsilon}^{*} \mathrm{e}^{-\mathrm{i} \boldsymbol{k} \cdot \boldsymbol{r}} a^{\dagger}\right)$, where $g=\left(2 V_{0} \omega\right)^{-1 / 2}$, with $V_{0}$ being the normalization volume of the photon field, and $\epsilon=$ $\epsilon_{x} \cos (\xi / 2)+\mathrm{i} \epsilon_{y} \sin (\xi / 2)$ being the polarization vector. The angle $\xi$ determines the degree of polarization, such that $\xi=\pi / 2$ corresponds to circular polarization and $\xi=0$ to linear polarization.

The transition matrix element, according to Guo, Åberg, and Crasemann (GAC) [9], is

$$
T_{f i}=\sum_{\mu\left(\mathcal{E}_{\mu}=\mathcal{E}_{i}\right)}\left\langle\Phi_{f}, n_{f}, 1\left(\left|\Psi_{\mu}, n_{s}^{\prime}\right\rangle+\left|\Psi_{\mu}, n_{s}^{\prime}\right\rangle^{\prime}\right)\right.
$$

$$
\times\left(\left\langle\Psi_{\mu}, n_{s}^{\prime}\left|+\left\langle\Psi_{\mu},\left.n_{s}^{\prime}\right|^{\prime}\right)\left(V+V^{\prime}\right)\right| \Phi_{i}, n_{i}, 0\right\rangle,\right.
$$

where

$$
\left|\Psi_{\mu}, n_{s}^{\prime}\right\rangle^{\prime}=\sum_{\mu^{\prime}}\left|\Psi_{\mu^{\prime}}, n_{s}^{\prime \prime}\right\rangle \frac{\left\langle\Psi_{\mu^{\prime}}, n_{s}^{\prime \prime}\left|V^{\prime}\right| \Psi_{\mu}, n_{s}^{\prime}\right\rangle}{\mathcal{E}\left(\mu, n_{s}^{\prime}\right)-\mathcal{E}\left(\mu^{\prime}, n_{s}^{\prime \prime}\right)}
$$

is the first-order correction to the wavefunction perturbed by the harmonic mode. Here, $n_{s}^{\prime}$ and $n_{s}^{\prime \prime}$ denote the spontaneously emitted photon number, $n_{i}$ the initial photon number of laser mode, and $n_{f}$ the final one. The index $\mu \equiv\{\boldsymbol{P} n\}$ characterizes a quantum-field Volkov state [14]:

$$
\begin{aligned}
\left|\Psi_{\mu}\right\rangle= & V_{e}^{-1 / 2} \sum_{l} \exp \left\{\mathrm{i}\left[\boldsymbol{P}+\left(u_{p}-l\right) \boldsymbol{k}\right] \cdot \boldsymbol{r}\right\} \\
& \times \mathcal{X}_{-l}\left(z_{c}, z_{s}\right)|n+l\rangle,
\end{aligned}
$$

which describes a nonrelativistic electron with momentum $\boldsymbol{P}$ moving in a field of photon number $n$, and the corresponding energy $\mathcal{E}_{\mu}=\boldsymbol{P}^{2} /\left(2 m_{e}\right)+\left(n+u_{p}+1 / 2\right) \omega$, where $V_{e}$ is the normalization volume of electron, $|n+l\rangle$ the Fock state of the laser photon mode, $l$ the number of transferred photons, $u_{p}=U_{p} / \omega$ the ponderomotive energy per laser photon energy. In latter text, we use $e_{b}$ instead of $I_{p} / \omega$, and $e_{b}^{\prime}=e_{b}+u_{p}$ for simplification. The phased Bessel function $X_{m}(z)$ and the generalized phased Bessel (GPB) functions [15] $\mathcal{X}_{l}\left(z_{c}, z_{s}\right)$ are defined as

$$
\begin{aligned}
& X_{m}(z)=J_{m}(|z|) \mathrm{e}^{\mathrm{i} \arg (z)}, \\
& \mathcal{X}_{j}\left(z_{c}, z_{s}\right)=\sum_{m} X_{j-2 m}\left(z_{c}\right) X_{m}\left(z_{s}\right),
\end{aligned}
$$

with $z_{c}=\left[2|e| \Lambda /\left(m_{e} \omega\right)\right] \boldsymbol{P} \cdot \boldsymbol{\epsilon}$ and $z_{s}=(1 / 2) u_{p} \boldsymbol{\epsilon} \cdot \boldsymbol{\epsilon}$.

In Eq. (5), the summing index $\mu^{\prime}$ means $\left\{\boldsymbol{P}^{\prime} n^{\prime}\right\}$. In Eq. (4), $n_{s}^{\prime}$ and $n_{s}^{\prime \prime}=0$ or 1 , denoting none or one harmonic photon, respectively. Treating the harmonic interaction as a perturbation, we obtain the reduced transition matrix element as a sum of the following three terms [13]:

$$
\begin{aligned}
T_{1} & =\sum_{\mu\left(\mathcal{E}_{\mu}=\mathcal{E}_{i}\right)}\left\langle\Phi_{f}, n_{f}, 1 \mid \boldsymbol{P} n, 1\right\rangle\left\langle\boldsymbol{P} n, 1\left|V^{\prime}\right| \Phi_{i}, n_{i}, 0\right\rangle, \\
T_{2} & =\sum_{\mu\left(\mathcal{E}_{\mu}=\mathcal{E}_{i}\right)}\left\langle\Phi_{f}, n_{f}, 1 \mid \boldsymbol{P} n, 1\right\rangle\left\langle\boldsymbol{P} n,\left.1\right|^{\prime} V \mid \Phi_{i}, n_{i}, 0\right\rangle, \\
T_{3} & =\sum_{\mu\left(\mathcal{E}_{\mu}=\mathcal{E}_{i}\right)}\left\langle\Phi_{f}, n_{f}, 1 \mid \boldsymbol{P} n, 0\right\rangle^{\prime}\left\langle\boldsymbol{P} n, 0|V| \Phi_{i}, n_{i}, 0\right\rangle,
\end{aligned}
$$

where the sum over $\mu$ can be performed as $\sum_{\mu} \equiv \sum_{\boldsymbol{P}_{n}}=$ $V_{e}^{-1}(2 \pi)^{3} \sum_{n} \int \mathrm{d}^{3} \boldsymbol{P}$.

According to the energy conservation between the initial state and the final state required by the scattering theory, we obtain the frequency condition for the harmonic

$$
\omega^{\prime}=E_{b f}-E_{b i}+\left(n_{i}-n_{f}\right) \omega .
$$


In the case when the excited photoelectron jumps back to the initial state, we have $\Phi_{f}=\Phi_{i}$ and $E_{b f}=E_{b i}$. Then Eq. (8) becomes

$$
\omega^{\prime}=\left(n_{i}-n_{f}\right) \omega \equiv q \omega .
$$

After a lengthy manipulation, we obtain explicit expressions for these three terms:

$$
\begin{aligned}
T_{1}= & \frac{|e| g^{\prime} \epsilon^{\prime *} .}{m_{e}(2 \pi)^{3}} \sum_{j \geqslant\left[e_{b}^{\prime}\right]+1} \int \mathrm{d}^{3} \boldsymbol{P} \Phi_{f}\left(\boldsymbol{P}^{\prime}\right)^{*} \Phi_{i}\left(\boldsymbol{P}^{\prime}+\boldsymbol{k}^{\prime}-q \boldsymbol{k}\right) \\
& \times \mathcal{X}_{-j}\left(z_{c}, z_{s}\right)\left\{\left(\boldsymbol{P}^{\prime}-q \boldsymbol{k}\right) \mathcal{X}_{-j-q}\left(z_{c}, z_{s}\right)^{*}\right. \\
& +|e|\left[\epsilon \Lambda \mathcal{X}_{-j-q+1}\left(z_{c}, z_{s}\right)^{*}\right. \\
& \left.\left.+\epsilon^{*} \Lambda \mathcal{X}_{-j-q-1}\left(z_{c}, z_{s}\right)^{*}\right]\right\},
\end{aligned}
$$

with

$$
\begin{aligned}
& P= \sqrt{2 m_{e} \omega\left(j-e_{b}^{\prime}\right)} \\
& \sum_{j \geqslant\left[e_{b}^{\prime}\right]+1} \int \mathrm{d}^{3} \boldsymbol{P}=\sum_{j \geqslant\left[e_{b}^{\prime}\right]+1}\left[2\left(j-e_{b}^{\prime}\right) m_{e}^{3} \omega^{3}\right]^{\frac{1}{2}} \int \mathrm{d} \Omega_{p}, \\
& \boldsymbol{P}^{\prime}=\boldsymbol{P}+\left(u_{p}-j+q\right) \boldsymbol{k}, \\
& T_{2}=\frac{|e| g^{\prime} \epsilon^{\prime *} \cdot \sum_{e}(2 \pi)^{3}}{m_{j^{\prime}} \geqslant\left[e_{b}^{\prime}\right]+1} \int \mathrm{d}^{3} \boldsymbol{P} \mathcal{X}_{-j^{\prime}}\left(z_{c}, z_{s}\right) \Phi_{f}\left(\boldsymbol{P}^{\prime \prime}\right)^{*} \\
& \quad \times \Phi_{i}\left(\boldsymbol{P}^{\prime \prime}-q \boldsymbol{k}+\boldsymbol{k}^{\prime}\right) \sum_{j} \frac{\left(u_{p}-j\right) \omega}{\Delta K E+\left(j-j^{\prime}\right) \omega} \\
& \quad \times \mathcal{X}_{-j}\left(z_{c}^{\prime}, z_{s}\right)^{*}\left\{\left(\boldsymbol{P}^{\prime \prime}+j^{\prime} \boldsymbol{k}\right) X_{j^{\prime}-j+q}\left(z_{k^{\prime}}\right)\right. \\
&+\frac{1}{2} \boldsymbol{k}\left[z_{c} X_{j^{\prime}-j-1+q}\left(z_{k^{\prime}}\right)+z_{c}^{*} X_{j^{\prime}-j+1+q}\left(z_{k^{\prime}}\right)\right. \\
&\left.+2\left(z_{s} X_{j^{\prime}-j-2+q}\left(z_{k^{\prime}}\right)+z_{s}^{*} X_{j^{\prime}-j+2+q}\left(z_{k^{\prime}}\right)\right)\right] \\
&+|e| \Lambda\left[\boldsymbol{\epsilon} X_{j^{\prime}-j-1+q}\left(z_{k^{\prime}}\right)\right. \\
&\left.\left.+\boldsymbol{\epsilon}^{*} X_{j^{\prime}-j+q+1}\left(z_{k^{\prime}}\right)\right]\right\},
\end{aligned}
$$

with

$$
\begin{aligned}
& P= \sqrt{2 m_{e} \omega\left(j^{\prime}-e_{b}^{\prime}\right)}, \\
& z_{c}^{\prime}= \frac{2|e| \Lambda}{m_{e} \omega} \boldsymbol{P}^{\prime} \cdot \boldsymbol{\epsilon}, \quad z_{k^{\prime}}=\frac{2|e| \Lambda}{m_{e} \omega} \boldsymbol{k}^{\prime} \cdot \boldsymbol{\epsilon}, \\
& \boldsymbol{P}^{\prime}= \boldsymbol{P}-\left(j^{\prime}-j+q\right) \boldsymbol{k}+\boldsymbol{k}^{\prime}, \quad \boldsymbol{P}^{\prime \prime}=\boldsymbol{P}+\left(u_{p}-j^{\prime}\right) \boldsymbol{k}, \\
& \Delta K E=\frac{\boldsymbol{P}}{m_{e}} \cdot\left[\left(j^{\prime}-j+q\right) \boldsymbol{k}-\boldsymbol{k}^{\prime}\right], \\
& T_{3}=\frac{|e| g^{\prime} \epsilon^{\prime *}}{m_{e}(2 \pi)^{3}} \sum_{j \geqslant\left[e_{b}^{\prime}\right]+1} \int \mathrm{d}^{3} \boldsymbol{P} \Phi_{f}\left(\boldsymbol{P}^{\prime \prime}+q \boldsymbol{k}-\boldsymbol{k}^{\prime}\right)^{*} \\
& \quad \times \mathcal{X}_{-j}\left(z_{c}, z_{s}\right)^{*} \Phi_{i}\left(\boldsymbol{P}^{\prime \prime}\right) \sum_{j^{\prime}} \mathcal{X}_{-j^{\prime}}\left(z_{c}^{\prime}, z_{s}\right) \\
& \quad \times \frac{\left(u_{p}-j\right) \omega}{\Delta K E+\left(j^{\prime}-j\right) \omega}\left\{\left(\boldsymbol{P}^{\prime \prime}+j \boldsymbol{k}\right) X_{j^{\prime}-j+q}\left(z_{k^{\prime}}\right)\right.
\end{aligned}
$$

$$
\begin{aligned}
& +\frac{1}{2} \boldsymbol{k}\left[z_{c} X_{j^{\prime}-j+q-1}\left(z_{k^{\prime}}\right)+z_{c}^{*} X_{j^{\prime}-j+q+1}\left(z_{k^{\prime}}\right)\right. \\
& \left.+2\left(z_{s} X_{j^{\prime}-j+q-2}\left(z_{k^{\prime}}\right)+z_{s}^{*} X_{j^{\prime}-j+q+2}\left(z_{k^{\prime}}\right)\right)\right] \\
& +|e| \Lambda\left[\epsilon X_{j^{\prime}-j+q-1}\left(z_{k^{\prime}}\right)\right. \\
& \left.\left.+\epsilon^{*} X_{j^{\prime}-j+q+1}\left(z_{k^{\prime}}\right)\right]\right\}
\end{aligned}
$$

with

$$
\begin{aligned}
& P=\sqrt{2 m_{e} \omega\left(j-e_{b}^{\prime}\right)}, \\
& \boldsymbol{P}^{\prime}=\boldsymbol{P}-\left(j-j^{\prime}-q\right) \boldsymbol{k}-\boldsymbol{k}^{\prime}, \quad \boldsymbol{P}^{\prime \prime}=\boldsymbol{P}+\left(u_{p}-j\right) \boldsymbol{k}, \\
& \Delta K E=\frac{\boldsymbol{P}}{m_{e}} \cdot\left[\left(j-j^{\prime}-q\right) \boldsymbol{k}+\boldsymbol{k}^{\prime}\right],
\end{aligned}
$$

where $z_{c}^{\prime}$ and $z_{k}^{\prime}$ are as defined earlier with noting that a defferent $\boldsymbol{P}^{\prime}$ from the previous one is involved.

The physical meanings of the sub-processes described in above rate formula can be understood by detailed inspection of the involved summation processes. For example, in the $T_{3}$ term, the index $j$ stands for the ionization photon number, and the harmonic order $q=j+\left(n-n_{f}\right)$. The difference $q-j=n-n_{f}$ stands for the extra photon absorptions when the harmonic order $q$ is high enough. Those extra photons do not participate the photon ionization process. They only participate, joining with ionization photons, the photon-mode up-conversion. This process can be called accompanying Roman effect, beside the photoionization effect.

This formula allows us to calculate HHG propagating in all directions. In our all-direction calculations, we find that the rate of HHG in any other direction is much less than that in the propagation direction of the laser beam. This fact also agrees with experimental observations [16]. In this paper, we only calculate spectra of high harmonics propagating in the laser beam direction.

In the direction $\boldsymbol{k}^{\prime} / / \boldsymbol{k}$, the three terms simplify as:

$$
\begin{aligned}
T_{1}= & \frac{|e| g^{\prime}\left(2 m_{e} \omega^{3}\right)^{1 / 2}}{(2 \pi)^{3}} \sum_{j \geqslant\left[e_{b}^{\prime}\right]+1}\left(j-e_{b}^{\prime}\right)^{1 / 2} \int \mathrm{d} \Omega \Phi_{f}(\boldsymbol{P})^{*} \\
& \times \Phi_{i}(\boldsymbol{P}) \mathcal{X}_{-j}\left(z_{c}, z_{s}\right)\left\{\epsilon^{\prime *} \cdot \boldsymbol{P} \mathcal{X}_{-j-q}\left(z_{c}, z_{s}\right)^{*}\right. \\
& +|e| \Lambda\left[\epsilon^{\prime *} \cdot \epsilon \mathcal{X}_{-j-q+1}\left(z_{c}, z_{s}\right)^{*}\right. \\
& \left.\left.+\epsilon^{\prime *} \cdot \epsilon^{*} \mathcal{X}_{-j-q-1}\left(z_{c}, z_{s}\right)^{*}\right]\right\}, \\
T_{2}= & \frac{|e| g^{\prime}\left(2 m_{e} \omega^{3}\right)^{1 / 2}}{(2 \pi)^{3}} \sum_{j^{\prime} \geqslant\left[e_{b}^{\prime}\right]+1}\left(j^{\prime}-e_{b}^{\prime}\right)^{1 / 2} \int \mathrm{d} \Omega_{\boldsymbol{P}} \Phi_{f}(\boldsymbol{P})^{*} \\
\times & \Phi_{i}(\boldsymbol{P}) \mathcal{X}_{-j^{\prime}}\left(z_{c}, z_{s}\right)\left\{\epsilon^{\prime *} \cdot \boldsymbol{P} \frac{u_{p}-j^{\prime}-q}{B q} \mathcal{X}_{-j^{\prime}-q}\left(z_{c}, z_{s}\right)^{*}\right. \\
& +|e| \Lambda\left[\epsilon^{\prime *} \cdot \epsilon \frac{u_{p}-j^{\prime}-q+1}{B(q-1)} \mathcal{X}_{-j^{\prime}-q+1}\left(z_{c}, z_{s}\right)^{*}\right. \\
& \left.\left.+\epsilon^{\prime *} \cdot \epsilon^{*} \frac{u_{p}-j^{\prime}-q-1}{B(q+1)} \mathcal{X}_{-j^{\prime}-q-1}\left(z_{c}, z_{s}\right)^{*}\right]\right\}, \quad(14)
\end{aligned}
$$




$$
\begin{aligned}
T_{3}= & \frac{|e| g^{\prime}\left(2 m_{e} \omega^{3}\right)^{1 / 2}}{(2 \pi)^{3}} \sum_{j \geqslant\left[e_{b}^{\prime}\right]+1}\left(j-e_{b}^{\prime}\right)^{1 / 2} \int \mathrm{d} \Omega_{\boldsymbol{P}} \Phi_{f}(\boldsymbol{P})^{*} \\
& \times \Phi_{i}(\boldsymbol{P}) \mathcal{X}_{-j}\left(z_{c}, z_{s}\right)^{*}\left\{\epsilon^{\prime *} \cdot \boldsymbol{P} \frac{u_{p}-j}{-B q} \mathcal{X}_{-j+q}\left(z_{c}, z_{s}\right)\right. \\
& +|e| \Lambda\left[\epsilon^{\prime *} \cdot \epsilon \frac{u_{p}-j}{-B(q-1)} \mathcal{X}_{-j+q-1}\left(z_{c}, z_{s}\right)\right. \\
& \left.\left.+\epsilon^{\prime *} \cdot \epsilon^{*} \frac{u_{p}-j}{-B(q+1)} \mathcal{X}_{-j+q+1}\left(z_{c}, z_{s}\right)\right]\right\},
\end{aligned}
$$

where $B \equiv 1-\boldsymbol{P} \cdot \boldsymbol{k} /\left(m_{e} \omega\right)$.

The final transition rate of HHG can be calculated by

$$
\left.\frac{\mathrm{d} W}{\mathrm{~d} \Omega_{\boldsymbol{k}^{\prime}}}\right|_{\boldsymbol{k}^{\prime} / / \boldsymbol{k}}=\frac{q \omega^{4} e^{2} m_{e}}{(2 \pi)^{8}}\left|\frac{T_{1}+T_{2}+T_{3}}{D}\right|^{2},
$$

where we use $D \equiv|e| g^{\prime}\left(2 m_{e} \omega^{3}\right)^{1 / 2} /(2 \pi)^{3}$ to replace the coefficients of $T_{1}, T_{2}$, and $T_{3}$.

\section{Numerical calculations and discussion}

With the transition rate formula, we are able to calculate the HHG spectra from all atoms. The atomic wave functions are calculated with Dirac-Hartree-Fock method [17].

In the following we show the main results from our calculations. We choose $\mathrm{Xe} 5 P_{3 / 2}$ as the sample atomic bound state, shined by linearly polarized laser light of wavelength $800 \mathrm{~nm}$. The laser beam intensity is chosen in four different values from $5 \times 10^{14}$ to $2 \times 10^{15} \mathrm{~W} / \mathrm{cm}^{2}$. Figure 1 shows the calculated HHG spectra which have a plateau and a clear cutoff structure.

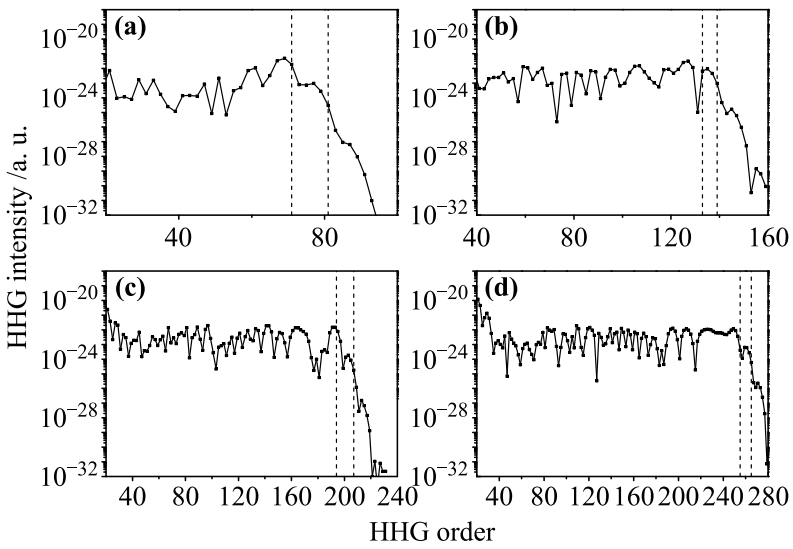

Fig. 1 HHG spectra generated from Xe atom driven by the laser pulses of wavelength $800 \mathrm{~nm}$ and intensities $0.5,1.0,1.5$ and $2.0 \times 10^{15} \mathrm{~W} / \mathrm{cm}^{2}$ from (a) to (d), respectively. The left dashed line and right dashed line in each subgraph refer to the cutoff orders from the cutoff law $q_{c} \hbar \omega=3.17 U_{p}+1.32 I_{p}$ and $q_{c} \hbar \omega=3.34 U_{p}+1.83 I_{p}$, respectively.

In these spectra, there are no even order harmonics. This feature can be understood directly from the parity property of GPB functions in these three terms $T_{1}, T_{2}$ and $T_{3}$ which are shown in Eqs. (13), (14) and (15). The nonvanishing of the integrals requires the integrants to be even functions. Now we change the momentum vector $\boldsymbol{P}$ to $-\boldsymbol{P}$ to test the parity property of the integrands. The phased Bessel and GPB functions have parity property [15]:

$$
\begin{aligned}
& X_{m}(-z)=(-1)^{m} X_{m}(z), \\
& \mathcal{X}_{j}\left(-z_{c}, z_{s}\right)=(-1)^{j} \mathcal{X}_{j}\left(z_{c}, z_{s}\right),
\end{aligned}
$$

with $z_{c}=\left[2|e| \Lambda /\left(m_{e} \omega\right)\right] \boldsymbol{P} \cdot \boldsymbol{\epsilon}$ and $z_{s}=(1 / 2) u_{p} \boldsymbol{\epsilon} \cdot \boldsymbol{\epsilon}$, we get $\mathcal{X}_{j}\left(-z_{c}, z_{s}\right)=(-1)^{j} \mathcal{X}_{j}\left(z_{c}, z_{s}\right)$. In Eq. (13), we get $\Phi_{f}(\boldsymbol{P})^{*} \Phi_{i}(\boldsymbol{P})=4 \pi\left|\Phi_{i}(|\boldsymbol{P}|)\right|^{2}$, and

$$
\begin{aligned}
\mathcal{X}_{-j} & \left(-z_{c}, z_{s}\right)\left\{\epsilon^{\prime *} \cdot(-\boldsymbol{P}) \mathcal{X}_{-j-q}\left(-z_{c}, z_{s}\right)^{*}\right. \\
& +|e| \Lambda\left[\epsilon^{\prime *} \cdot \epsilon \mathcal{X}_{-j-q+1}\left(-z_{c}, z_{s}\right)^{*}\right. \\
& \left.\left.+\epsilon^{\prime *} \cdot \epsilon^{*} \mathcal{X}_{-j-q-1}\left(-z_{c}, z_{s}\right)^{*}\right]\right\}, \\
= & (-1)^{(-q+1)} \mathcal{X}_{-j}\left(z_{c}, z_{s}\right)\left\{\epsilon^{\prime *} \cdot \boldsymbol{P} \mathcal{X}_{-j-q}\left(z_{c}, z_{s}\right)^{*}\right. \\
& +|e| \Lambda\left[\epsilon^{\prime *} \cdot \epsilon \mathcal{X}_{-j-q+1}\left(z_{c}, z_{s}\right)^{*},\right. \\
& \left.\left.+\epsilon^{\prime *} \cdot \epsilon^{*} \mathcal{X}_{-j-q-1}\left(z_{c}, z_{s}\right)^{*}\right]\right\} .
\end{aligned}
$$

In the integration over $\boldsymbol{P}$, the integrand is an odd function when $\mathrm{q}$ is even due to the factor $(-1)^{-q+1}$. Noting the denominator $B \equiv 1-\boldsymbol{P} \cdot \boldsymbol{k} /\left(m_{e} \omega\right) \approx 1$ in the nonrelativistic limit, with the same analysis in Eqs. (14) and (15), the integrand is odd when q is even, which leads to the vanishing of the integral.

We also compute HHG spectra for $\mathrm{He}, \mathrm{Ne}, \mathrm{Ar}$, and $\mathrm{Kr}$, shined by linearly polarized laser light of wavelength $800 \mathrm{~nm}$ and intensity $1.5 \times 10^{15} \mathrm{~W} / \mathrm{cm}^{2}$. These spectra show some common features: a well formed plateau and a sharp cutoff structure. The calculated spectra are presented in Fig. 2.

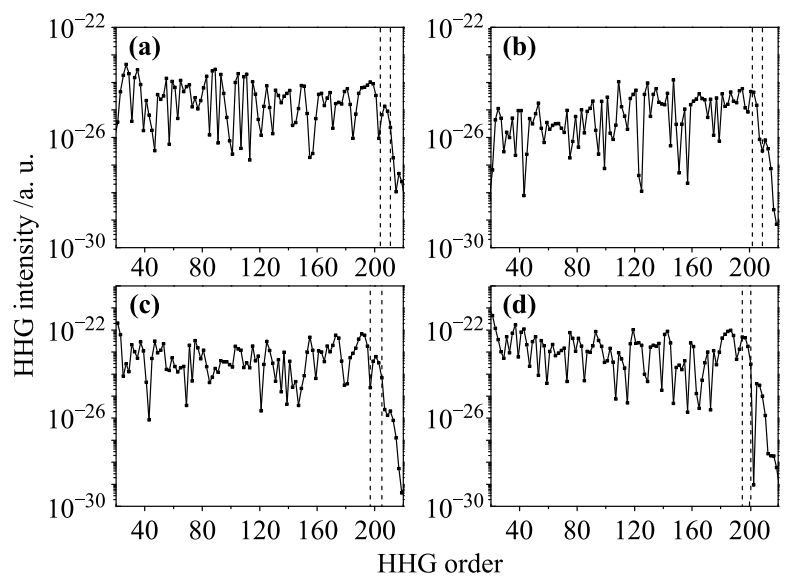

Fig. 2 HHG spectra generated from (a) He, (b) Ne, (c) Ar, and (d) $\mathrm{Kr}$ atom driven by the laser pulses of wavelength $800 \mathrm{~nm}$ and intensities $1.5 \times 10^{15} \mathrm{~W} / \mathrm{cm}^{2}$. The left dashed line and right dashed line in each subgraph refer to the cutoff orders from the cutoff law $q_{c} \hbar \omega=3.17 U_{p}+1.32 I_{p}$ and $q_{c} \hbar \omega=3.34 U_{p}+1.83 I_{p}$, respectively. 
There are many discussions on the plateau of the spectra in literatures. In this paper, we focus on the discussion of the cutoff property of the spectra.

In a very recent theoretical work [18], we obtained an expression of the cutoff law for HHG spectra, $q_{c} \omega=$ $3.34 U_{p}+1.83 I_{p}$, where we only used the cutoff property of ordinary Bessel functions and the Einstein photoelectric law in the multiphoton case, without any spectra calculation. The data from a very recent experiment [8] support the new cutoff law. Now we have the transition rate formula to calculate the HHG spectra. Those spectra obtained from our rate calculation will offer a strong numerical check to the new cutoff law, also to the previously well-accepted cutoff laws. In Fig. 3, the circle points show the cutoff orders from our spectra calculation. The sample atomic state to ionize is Xe $5 P_{3 / 2}$, shined by linearly polarized laser light of wavelength $800 \mathrm{~nm}$. We choose 16 different beam intensities from $5 \times 10^{14}$ to $2 \times 10^{15}$ $\mathrm{W} / \mathrm{cm}^{2}$. The best fitting line of these scattered points coincides well with the line $3.34 U_{p}+1.83 I_{p}$.

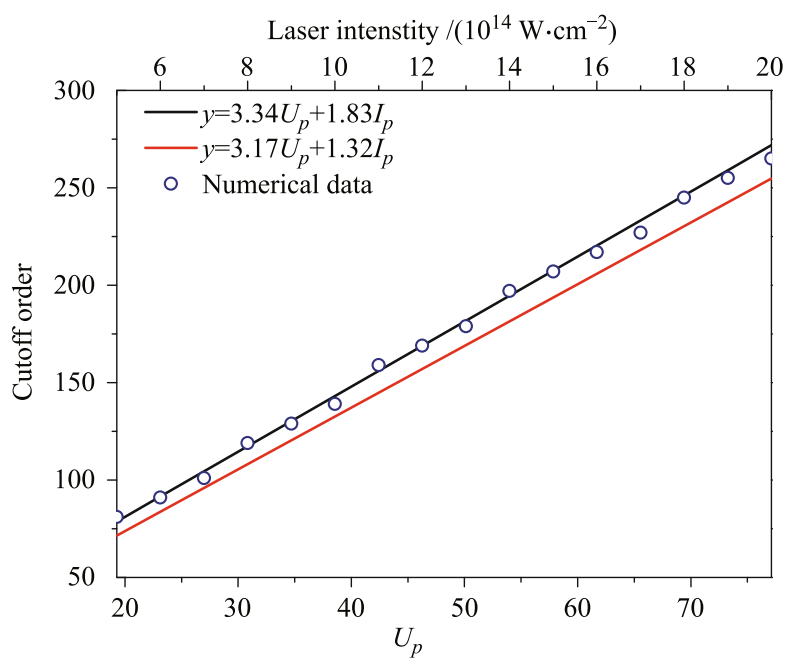

Fig. 3 The circle points refer to the cutoff orders obtained from the HHG spectra calculation. The best fitting line to the circle points coincides with the line $3.34 U_{p}+1.83 I_{p}$.

Why the cutoff order predicted by our theory is higher than that predicted by the " 3.17 " law? To answer this question, we need to look into the details in our formulas for the calculation. In a good quantum mechanic theory, all kinds of conservation laws should be automatically derived from the theory. If a transition subprocess is mathematically allowed, it must meet all kinds of conservation laws. Thus, one has to include any possible transition subprocess as an open channel in the calculation. In our formula, the participating photons for HHG are not only the ionization photons, but also some nonionization photons which participate the photon-mode up-conversion. The process for a photoelectron absorbing non-ionization photons in photon-mode up-conversions may be called the accompanying Raman effect. It is the accompanying Raman effect which makes the cutoff order of HHG greater than the one predicted by semiclassical theories. The laser photons to participate the photon-mode up-conversion are not only those ionization photons which show up in the Einstein's photo-ionization law, but also some non-ionization photons. In a full quantum mechanical treatment, all possible transition channels must occur if they are not ruled out by mathematical restrictions which embody all kinds of conservation laws in physics. The absorbed laser photons to participate the photon-mode up-conversion are from both, the photo-ionization effect and the accompanying Raman effect.

\section{Summary}

The transition rate formula for HHG is strictly derived from the NPQED scattering theory. This formula allows us to calculate the HHG rates for different atoms in laser beams of arbitrary intensities and arbitrary wavelengths. Applying the transition rate formula, we calculate the HHG spectra for $\mathrm{He}, \mathrm{Ne}, \mathrm{Ar}, \mathrm{Kr}$, and Xe atoms. These applications can be used for future studies of HHG spectra.

According to the calculated spectra, we study the cutoff properties of HHG. In the spectra calculation, we neither use the commonly accepted cutoff law $[3,4]$ $q_{c} \hbar \omega=3.17 U_{p}+1.32 I_{p}$, nor the one derived recently [18] $q_{c} \hbar \omega=3.34 U_{p}+1.83 I_{p}$. Thus, the spectra calculation presented here offers an independent reference to different cutoff laws.

Acknowledgements This work was partly supported by the National Natural Science Foundation of China (Grant Nos. 11004060, 11027403, and 51132004). D.-S. Guo is partially supported by the Zijiang Program of East China Normal University and the Guest Professor Program of Peking University. Jingtao Zhang is partially supported by the National Natural Science Foundation of China (Grant Nos. 11174304 and 61078080), and the National Basic Research Program of China (973 Program) (Grant Nos. 2010CB923203 and 2011CB808103).

\section{References}

1. A. Mcpherson, G. Gibson, H. Jara, U. Johann, T. S. Luk, I. A. McIntyre, K. Boyer, and C. K. Rhodes, Studies of multiphoton production of vacuum-ultraviolet radiation in the rare gases, J. Opt. Soc. Am. B 4(4), 595 (1987)

2. J. T. Zhang and D.-S. Guo, Spectral minimum and giant enhancement in photoelectron spectra from xenon atoms driven by intense midinfrared laser fields, Phys. Rev. Lett. 
110(6), 063002 (2013)

3. P. B. Corkum, Plasma perspective on strong field multiphoton ionization, Phys. Rev. Lett. 71(13), 1994 (1993)

4. M. Lewenstein, P. Balcou, M. Y. Ivanov, A. L'Huillier, and P. B. Corkum, Theory of high-harmonic generation by lowfrequency laser fields, Phys. Rev. A 49(3), 2117 (1994)

5. J. Gao, F. Shen, and J. G. Eden, Quantum electrodynamic treatment of harmonic generation in intense optical fields, Phys. Rev. Lett. 81(9), 1833 (1998)

6. J. Gao, F. Shen, and J. G. Eden, Interpretation of highorder harmonic generation in terms of transitions between quantum Volkov states, Phys. Rev. A 61(4), 043812 (2000)

7. L. H. Gao, X. F. Li, P. M. Fu, R. R. Freeman, and D.-S. Guo, Nonperturbative quantum electrodynamics theory of high-order harmonic generation, Phys. Rev. A 61(6), 063407 (2000)

8. T. Popmintchev, M. Chen, D. Popmintchev, P. Arpin, S. Brown, S. Alisauskas, G. Andriukaitis, T. Balciunas, O. D. Mücke, A. Pugzlys, A. Baltuska, B. Shim, S. E. Schrauth, A. Gaeta, C. Hernandez-Garcia, L. Plaja, A. Becker, A. JaronBecker, M. M. Murnane, and H. C. Kapteyn, Bright coherent ultrahigh harmonics in the keV X-ray regime from midinfrared femtosecond lasers, Science 336(6086), 1287 (2012)

9. D.-S. Guo, T. Åberg, and B. Crasemann, Scattering theory of multiphoton ionization in strong fields, Phys. Rev. A
40(9), 4997 (1989)

10. D.-S. Guo, Exact wave functions for atomic electron interacting with photon fields, Front. Phys. 8(1), 39 (2013)

11. D.-S. Guo, J. T. Zhang, Z. R. Sun, J. T. Wang, J. Gao, Z.-W. Sun, and R. R. Freeman, Even-odd harmonics generated from above-threshold ionization, Front. Phys. 9(1), 69 (2014)

12. D. M. Volkov, On a class of solutions of the Dirac equation, Z. Phys 94, 250 (1935)

13. J. Gao, D.-S. Guo, and Y.-S. Wu, Resonant above-threshold ionization at quantized laser intensities, Phys. Rev. A 61(4), 043406 (2000)

14. D.-S. Guo and G. W. Drake, Stationary solutions for an electron in an intense laser field (I): Single-mode case, J. Phys. A: Math. Gen. 25(11), 3383 (1992)

15. X. Hu, H. X. Wang, and D.-S. Guo, Phased bessel functions, Can. J. Phys. 86(7), 863 (2008)

16. J. Peatross and D. D. Meyerhofer, Intensity-dependent atomic-phase effects in high-order harmonic generation, Phys. Rev. A 52(5), 3976 (1995)

17. W. R. Johnson, Atomic Structure Theory, 2007: Springer

18. D.-S. Guo, C. Yu, J. T. Zhang, J. Gao, Z.-W. Sun, and Z. R. Sun, On the cutoff law of laser high harmonic spectra, Front. Phys. 10, 103201 (2015) 